Journal of Computational Acoustics, Vol. 25, No. 2 (2017) 1792001 (2 pages)

(C) IMACS

DOI: 10.1142/S0218396X17920011

\title{
Erratum
}

\section{A Numerical Model of an Acoustic Metamaterial Using the Boundary Element Method Including Viscous and Thermal Losses}

\author{
[Journal of Computational Acoustics, Vol. 25 (2017), DOI: 10.1142/S0218396X17500060] \\ V. Cutanda Henríquez*, P. Risby Andersen ${ }^{\dagger}$ and J. Søndergaard Jensen ${ }^{\ddagger}$ \\ Centre for Acoustic-Mechanical Micro Systems \\ Technical University of Denmark, Ørsteds Plads \\ Building 352, DK-2800, Kgs. Lyngby, Denmark \\ *vcuhe@elektro.dtu.dk \\ ${ }^{\dagger}$ prand@elektro.dtu.dk \\ ${ }^{\ddagger} j$ json@elektro.dtu.dk \\ P. Møller Juhl \\ Marsk Mc-Kinney Møller Institute \\ University of Southern Denmark \\ Campusvej 55, DK-5230 Odense M, Denmark \\ pmjuhl@mmmi.sdu.dk \\ J. Sánchez-Dehesa \\ Wave Phenomena Group, Department of Electronic Engineering \\ Universitat Politècnica de València \\ Camino de Vera s.n. (building 7F), ES-46022 Valencia, Spain \\ jsanched@upv.es
}

Received 17 March 2017

Published 23 March 2017

One of the curves in Fig. 5 of the published paper should be modified. It corresponds to the transmittance measurement results. The results were obtained from a previous publication (Ref. 17 in the published paper), but the data was incorrectly squared.

Figure 1 below is a new version of Fig. 5 in the original paper, where the measurement curve has been plotted without squaring. The measurement plot is now closer to the transmittance curves calculated using the Finite Element Method and the Boundary Element Method with losses. 
V. Cutanda Henríquez et al.

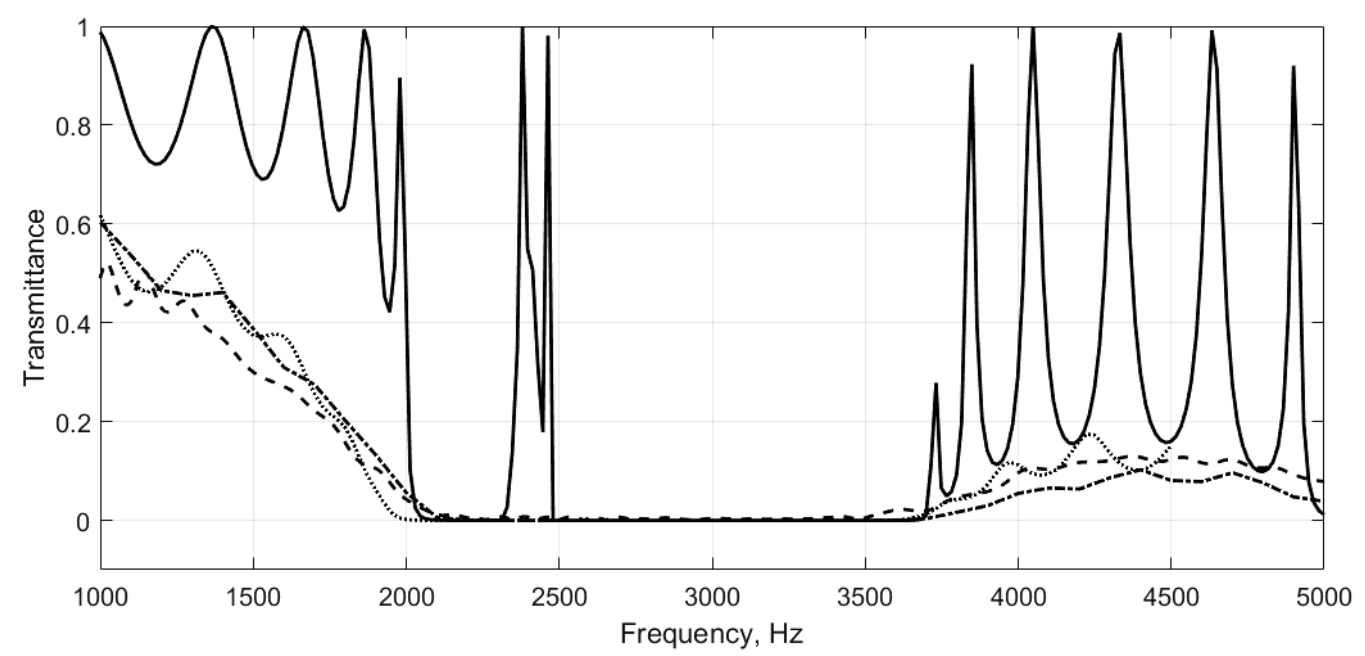

Fig. 1. Transmittance, obtained at the receiving end of the setup. Solid line, FEM model without losses; dotted line, FEM model with losses; dash-dotted line, BEM model with losses; dashed line, measurements by Graciá-Salgado et al. 